\title{
NEW DEVELOPMENTS IN HPGe DETECTORS FOR HIGH RESOLUTION DETECTION*
}

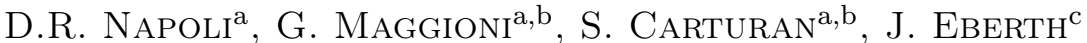

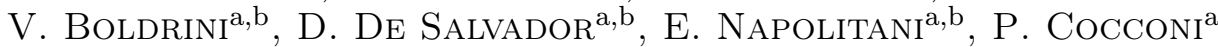

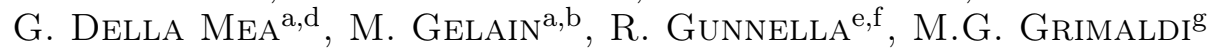 \\ M. Loriggiola ${ }^{\mathrm{a}}$, G. Mariotto ${ }^{\mathrm{a}, \mathrm{h}}, \mathrm{N}$ Pinto $^{\mathrm{e}, \mathrm{f}}, \mathrm{W} \cdot$ RAniero $^{\mathrm{a}}$

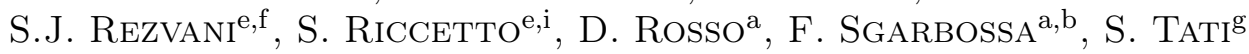 \\ ${ }^{a}$ INFN, Laboratori Nazionali di Legnaro, 35020 Legnaro (PD), Italy \\ ${ }^{\mathrm{b}}$ Dept. of Physics and Astronomy, University of Padova, 35131 Padova, Italy \\ ${ }^{\mathrm{C} I n s t i t u t ~ f u ̈ r ~ K e r n p h y s i k, ~ U n i v e r s i t a ̈ t ~ z u ~ K o ̈ l n, ~} 50937$ Köln, Germany \\ ${ }^{\mathrm{d}}$ Dept. of Industrial Engineering, University of Trento, 38123 Trento, Italy \\ eINFN, Section of Perugia, 06123 Perugia, Italy \\ ${ }^{\mathrm{f}}$ School of Science and Technology, University of Camerino, 62032 Camerino, Italy \\ ${ }^{g}$ Dept. of Physics and Astronomy, University of Catania, 95123 Catania, Italy \\ ${ }^{\mathrm{h}}$ Dept. of Computer Science, University of Verona, 37134 Verona, Italy \\ ${ }^{\mathrm{i}}$ Dept. of Physics and Geology, University of Perugia, 06123 Perugia, Italy
}

\section{(Received January 17, 2017)}

High-Purity Germanium (HPGe) detectors continue to be a fundamental tool in nuclear gamma spectroscopy. The tracking of the gamma interactions inside the HPGe crystals is opening a new era in the use of these detectors for both basic science and applications, but they have also shown that new R\&D is necessary for the production of even better and more reliable highly segmented detectors. In this work, we present recent results obtained in the framework of a multidisciplinary research program in HPGe detector technologies and we discuss the influence of these studies on the use of HPGe detectors.

DOI:10.5506/APhysPolB.48.387

\section{Introduction}

Gamma detectors made from hyperpure Ge crystals (HPGe) are still the best solution for high resolution gamma spectroscopy [1, 2]. In addition, in the last years, a new generation of gamma tracking detectors (i.e. AGATA [3]) has been developed by using segmented HPGe detectors with digital electronics and pulse-shape analysis techniques.

* Presented at the Zakopane Conference on Nuclear Physics "Extremes of the Nuclear Landscape", Zakopane, Poland, August 28-September 4, 2016. 
With the precise energy and three-dimensional position of each one of the interaction points of the Compton scattered gamma inside of the HPGe crystal and by means of sophisticated gamma-ray tracking algorithms, it is possible to reconstruct the sequence and trajectory of the successive Compton scattered gamma rays. With this information, it has been shown that it is feasible to determine the full energy, the first interaction point and the incident direction of the gamma rays that hit the highly segmented HPGe detector $[4,5]$.

For a better gamma tracking, it is necessary to establish all the interaction points and for that purpose, it is quite important to reduce the amount of regions inside the crystal where poor charge collection could give false inputs to the determination of the Compton scattering trajectories. Then, additional R\&D is still needed to produce better and more reliable detectors to achieve a wider use of gamma tracking technologies in other fields.

The Legnaro National Laboratories (LNL) has a long tradition in nuclear spectroscopy studies. Many gamma-ray arrays have been operated at LNL in the last 30 years, since the installation of MIPAD in 1987 [6]. In 1991, we have designed and installed GASP, a gamma array composed of $40 \mathrm{HPGe}$ detectors, that have been run since 1992 [7] till 2012. During the same period, we have installed other gamma arrays at LNL in collaboration with institutes of all Europe, like EUROBALL [8-10] in 1997-1998, CLARA [11] from 2004 to 2008 and recently, after the online gamma tracking demonstration phase, we have performed the first physics campaign with AGATA in the period of 2009-2011 [3, 12]. The experience accumulated in these years spurred us to start a multidisciplinary research program for developing new technologies for the fabrication of the next generation of highly segmented HPGe detectors for AGATA.

\section{2. $\mathrm{R} \& \mathrm{D}$ in HPGe detectors}

\subsection{HPGe gamma detectors}

An HPGe detector is a diode produced from an extremely pure Ge single crystal with impurity concentration around $10^{10}$ atoms $/ \mathrm{cm}^{3}$. The diode is reverse biased in order to achieve the complete charge depletion of the Ge crystal volume and the surface in-between the $n$ and $p$ contacts needs to be passivated to reduce the leakege currents that could mask the charges collected after a gamma interaction. As a result of gamma-ray interaction with the detector, electrical charges are created and driven to the contacts by the electric field. The intrinsic surface between these $n$ and $p$ contacts is the most critical part and must be passivated in order to remove electronic states in the Ge band gap (electrical passivation) and to prevent the surface from reacting with the atmosphere (chemical passivation) [1, 2, 13]. 
For the new gamma tracking technologies, where each gamma interaction inside the crystal gives important information for the determination of the properties of the incident gamma ray, it is necessary to establish the role of the surfaces treatments in the creation of dead layers that could deteriorate the performance of the gamma tracking detectors [1].

In parallel, in the last years, the study of Ge surface passivation has been steadily growing due to the renewed interest for microelectronics industrial applications because Ge has a higher charge mobility than $\mathrm{Si}$ (a factor of 3) and can be processed at a lower temperature (for example, Ge melts at $1210 \mathrm{~K}$ instead of $1683 \mathrm{~K}$ for $\mathrm{Si}$ ) [14].

In the frame of this research, several passivation routes have been proposed and studied also by our group for HPGe detector developments [15-21].

\subsection{HPGe passivation}

Several surface treatments have been characterized and the chemical composition of the newly formed monolayers has been investigated with regards to the nature of chemical bonding with Ge atop atoms by using Fourier Transform Infrared Spectrometry (FTIR) and X-ray Photoelectron Spectroscopy (XPS) in order to investigate, for example, the formation of $\mathrm{Ge}-\mathrm{H}$ chemical bonds and the stability of each surface after air exposure [18].

Figure 1 shows the XPS spectra of the differently passivated Ge surfaces: for each sample, the Ge2p peak is simulated by using a different peak deconvolution, which highlights the formation of surface bonds of Ge atoms with hydrogen, oxygen or sulphur depending on the treatment. In the case of $\mathrm{S}$ passivation, the Ge-S bond termination is also confirmed.

For all these methods, we have obtained good insulating properties under the application of high reverse voltage [18] and with the most promising candidates, we have developed the HPGe detectors that will be treated in the next section.

\subsection{Passivation properties}

To test the properties of methanol (Ge_QM), sulphur (Ge_S) and two different $H$-terminated (Ge_H10 and Ge_H50) passivations, we have prepared with them planar detectors $[15,16]$. These different passivation routes have been applied on the same cylindrical $n$-type bulk crystal in order to assure that the observed differences were related only to the surface treatments and not to bulk properties of different Ge crystals.

For these studies, we prepared a cylindrical planar detector of $21 \mathrm{~mm}$ height and $39 \mathrm{~mm}$ of diameter. The $p^{+}$contact was obtained by $\mathrm{B}$ implantation on one of the planar faces of the cylinder and the $n^{+}$contact by 

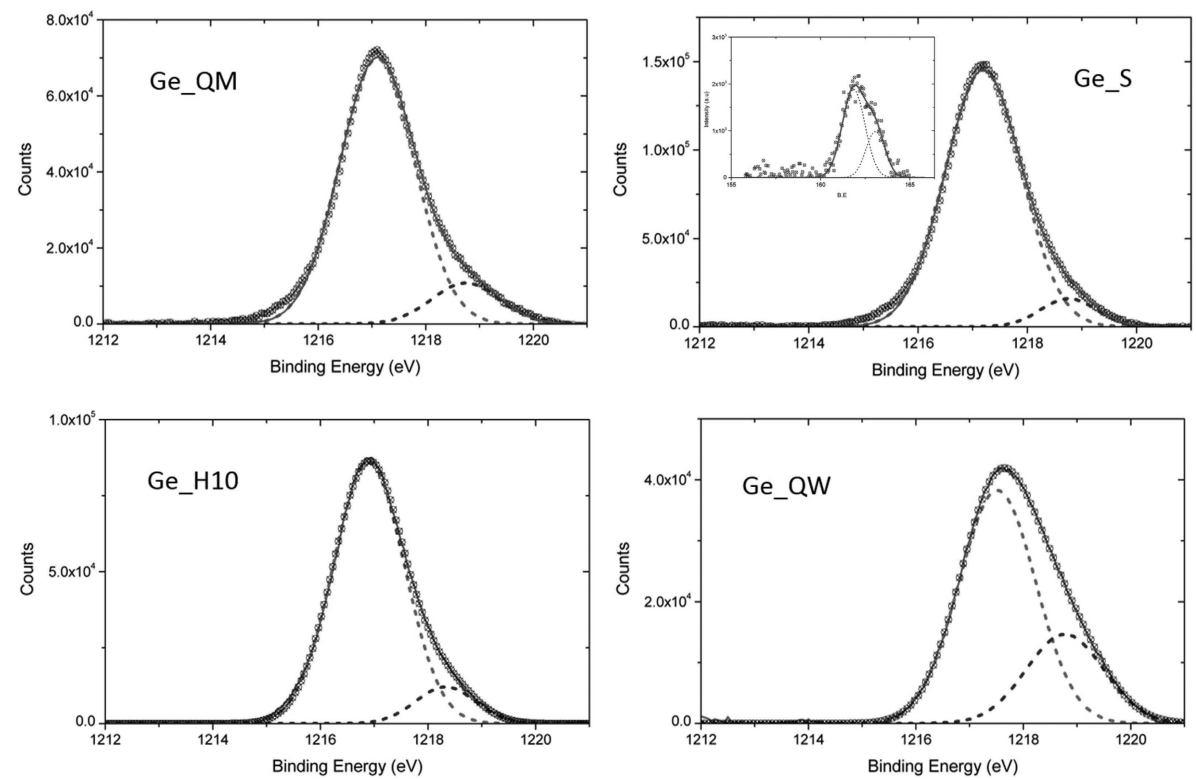

Fig. 1. XPS spectra of Ge2p peak, with corresponding peak deconvolution, for some of the wet treatments of the Ge surface cited in Ref [18]: methanol (Ge_QM), sulphur (Ge_S) the H-terminated (Ge_H10) and the Bidistilled Water Quenched (Ge_QW) passivations.

Li diffusion on the opposite face of the crystal. Both contacts were covered with Kapton during the different passivations treatments, which have been applied on the lateral cylindrical surface.

The performance of the dielectric layer has been determined directly from $\mathrm{I}-\mathrm{V}$ measurements of the HPGe diode, obtaining currents lower than $30 \mathrm{pA}$ at $1100 \mathrm{~V}$ for all the passivations $[15,16]$.

\subsection{HPGe detector properties}

For each detector, we have measured both the bulk properties of the detector (i.e. resolution, efficiency, counting rate vs. applied voltage, depletion voltage) and carried out an accurate scanning of the passivated surface with a low-energy gamma source.

For the determination of the detector properties, standard calibration gamma sources of ${ }^{60} \mathrm{Co},{ }^{152} \mathrm{Eu}$ and ${ }^{241} \mathrm{Am}$ were placed in front of the endcap of the cryostat, illuminating first the B-doped face of the detector.

The scanning of the passivated surface with a low-energy collimated ${ }^{241} \mathrm{Am}$ gamma source (59.5 keV) (figure 2) shows up that the surface passivation gives rise to a dead layer below the intrinsic Ge surface (figure 3), whose thickness and distribution are strongly dependent on the passivation type $[15,16]$. 


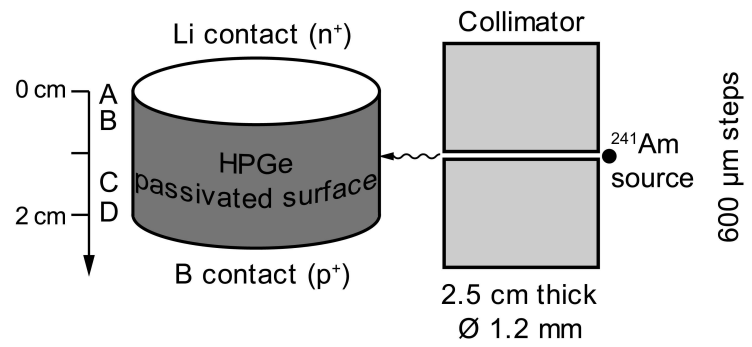

Fig. 2. Simplified scheme of the scanning setup. The collimator and the ${ }^{241} \mathrm{Am}$ gamma source can move along the passivated side of the crystal from the $n^{+}$contact to the opposite $p^{+}$contact in $0.6 \mathrm{~mm}$ steps [20].

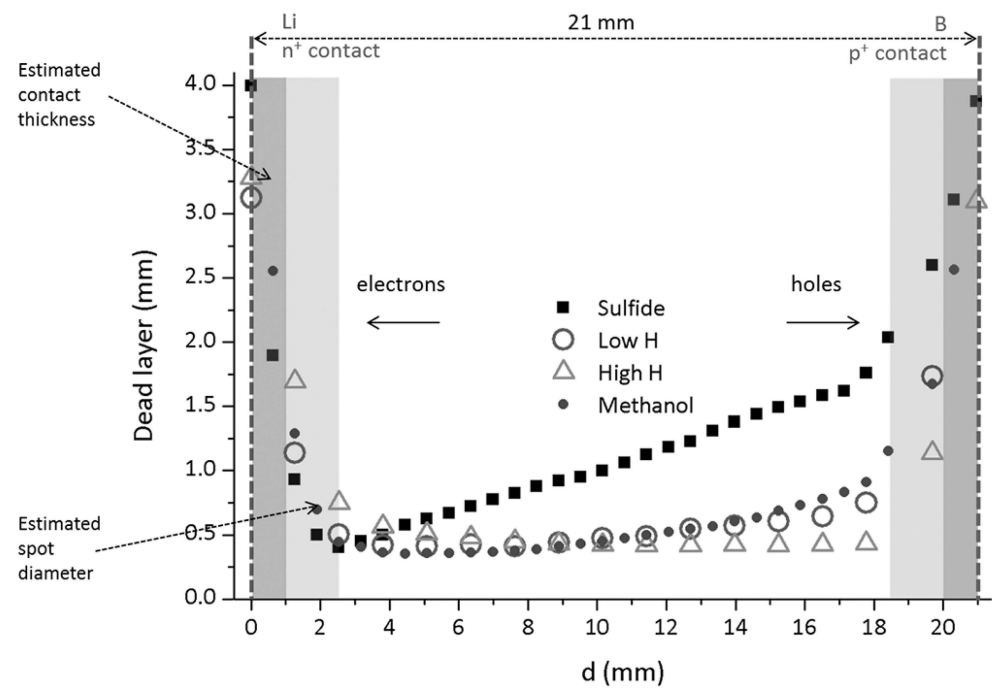

Fig. 3. Thickness of the dead layer as a function of the position of the ${ }^{241} \mathrm{Am}$ gamma source for different passivations [15].

As can be seen in figure 4, the active volume of the detector, estimated by the measured dead layer, is affected by the passivation type similarly to some bulk detector properties such as: the peak-to-Compton ratio, the detector efficiency and the plateau in the counting rate, when plotted as a function of the applied voltage. On the other hand, the optimal energy resolution was almost the same for all the passivations but at different voltages (see Table I). 

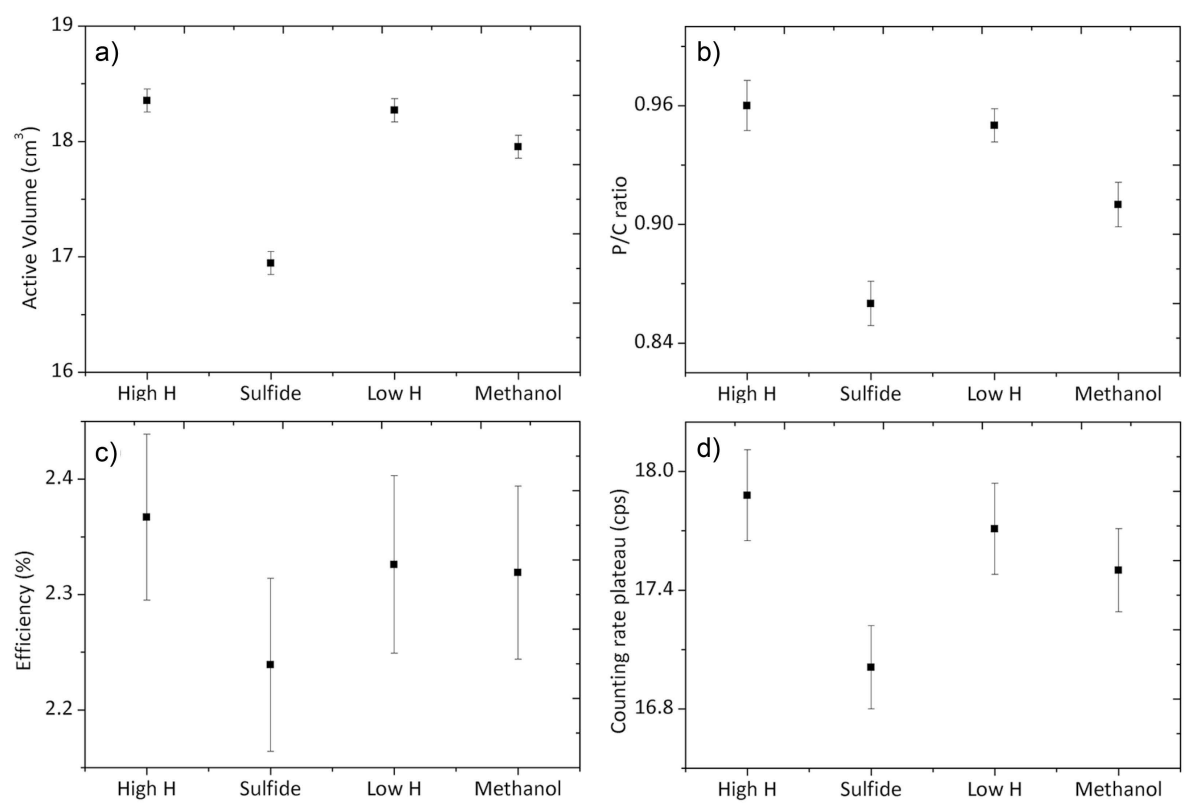

Fig. 4. For each one of the different passivations studied, we have determined: (a) the estimated active volume of the detector, calculated taking into account the measured dead layers shown in figure 3; (b) the peak-to-Compton ratio; (c) the detector efficiency, and (d) the counting rate at the plateau of the rate vs. voltage plot (from Ref. [15]).

TABLE I

The best resolution achieved for each detector and the corresponding bias voltage.

\begin{tabular}{l|c|c}
\hline \hline Detector Passivation & Resolution & Voltage \\
\hline Methanol & $1.80 \mathrm{keV}$ & $2000 \mathrm{~V}$ \\
Sulphur & $1.76 \mathrm{keV}$ & $2500 \mathrm{~V}$ \\
Low H & $1.74 \mathrm{keV}$ & $1500 \mathrm{~V}$ \\
High H & $1.73 \mathrm{keV}$ & $1500 \mathrm{~V}$
\end{tabular}

\section{Summary}

In the framework of an R\&D program in HPGe detector technologies, we have established the influence of the passivation in the creation of dead layers that can strongly affect the performance of gamma tracking detectors. Several passivation techniques have been applied to the same planar HPGe detector and their effects on the detector properties have been investigated. 
If one excludes the sulphur termination, the comparison between the different passivation methods tested in this work highlights that their effects on the detector properties are similar: the passivation gives rise to a dead layer inside the detector in the proximity of the passivated surface. As compared to the commercial passivations [1], the dead layers produced by the tested passivations are thinner and their thickness is more homogeneous. The detector with the thinnest average dead layer is the high- $H$-terminated one. Instead, the sulphur termination gives rise to a thicker average dead layer which reduces the active volume; moreover, the thickness of the dead layer is not constant and strongly increases going from the $n^{+}$to the $p^{+}$contact. The lateral scan measurements with the low-energy collimated source allowed to highlight the electrical nature of the passivated surfaces: all the passivations gave rise to an $n$-type surface, except for the high- $H$ termination, which produced a slightly $p$-type surface. Differences in photopeak counting rate could be explained with the hypothesis of weak or distorted field regions, where full electric charge collection is not achieved, because charges recombine or are driven elsewhere. To solve the problems related with the stability of these passivation methods, a protective coating has been developed [20]. The coating showed excellent properties of stability under the normal operational conditions for an in-beam used gamma detector, i.e. thermal cycles and successive annealings to recover neutron damage on the Ge crystal. Experimental results obtained with detectors protected by this new coating will be published in a forthcoming paper.

This work has been partially funded under the $7^{\text {th }}$ Framework Program FP7/2007-2013, grant agreement No. 262010 - ENSAR (INDESYS JRA) and under the PSeGe Joint Research Activity of the ENSAR2 framework for European research in nuclear physics, grant agreement No. 654002 of the Horizon 2020.

\section{REFERENCES}

[1] J. Eberth, J. Simpson, Prog. Part. Nucl. Phys. 60, 283 (2008).

[2] K. Vetter, Annu. Rev. Nucl. Part. Sci. 57, 363 (2007).

[3] S. Akkoyun et al., Nucl. Instrum. Methods Phys. Res. A 668, 26 (2012).

[4] R.M. Lieder et al., Prog. Part. Nucl. Phys. 46, 399 (2001).

[5] R.M. Lieder et al., Nucl. Phys. A 682, 279c (2001).

[6] G. Lobianco, Prog. Part. Nucl. Phys. 28, 487 (1992).

[7] D. Bazzacco et al., Phys. Lett. B 309, 235 (1993).

[8] J. Simpson, Z. Phys. A 358, 139 (1997). 
[9] C. Rossi Alvarez, Nuovo Cim. A 111, 601 (1998).

[10] S. Tormanen et al., Phys. Lett. B 454, 8 (1999).

[11] A. Gadea et al., Eur. Phys. J. A 20, 193 (2004).

[12] A. Gadea et al., Nucl. Instrum. Methods Phys. Res. A 654, 88 (2011).

[13] C. Fleischmann et al., J. Mater. Chem. C 1, 4105 (2013).

[14] P.W. Loscutoff, S.F. Bent, Annu. Rev. Phys. Chem. 57, 467 (2006).

[15] G. Maggioni et al., Eur. Phys. J. A 51, 141 (2015).

[16] D.R. Napoli et al., AIP Conf. Proc. 1753, 070004 (2016).

[17] G. Maggioni et al., Appl. Surf. Sci. 393, 119 (2017).

[18] S. Carturan et al., Mater. Chem. Phys. 161, 116 (2015).

[19] V. Boldrini et al., Appl. Surf. Sci. 392, 1173 (2017).

[20] M. Gelain et al., PoS X LASNPA, 042 (2014).

[21] N. Pinto et al., J. Non-Cryst. Solids 452, 280 (2016). 